\title{
Professionalisierung von Lehramtsstudierenden für inklusiven naturwissenschaftlichen Unterricht
}

\section{Daniela Egger, Sarah Brauns, Katja Sellin, Matthias Barth \& Simone Abels}

Journal für Psychologie, 27(2), 50-70

https://doi.org/10.30820/0942-2285-2019-2-50

www.journal-fuer-psychologie.de

\section{Zusammenfassung}

Durch die Ratifizierung der UN-Behindertenrechtskonvention (United Nations 2006, Artikel 24) hat sich Deutschland verpflichtet »Bildung für alle«(UNESCO 2015) im deutschen Schulsystem möglich zu machen. In der Lehrer*innenbildung stellen sich zwei Herausforderungen für die Fachdidaktiken: Erstens ist der Schulunterricht in verschiedene Fächer aufgegliedert, die jeweils eigene Fachinhalte aufweisen. Diese Inhalte müssen entlang fachdidaktischer Prinzipien inklusiv aufbereitet werden. Zweitens steigt die Komplexität der Fachinhalte vom Übergang der Grundschule in die Sekundarstufe deutlich an, sodass es für Lehrkräfte immer schwieriger wird, auf heterogene Lernvoraussetzungen angemessen einzugehen (Musenberg und Riegert 2015, 5). Die Professionalisierung von Lehrkräften muss gezielt auf diese Herausforderungen reagieren und Lehramtsstudierende nachhaltig auf ihr Tätigkeitsfeld vorbereiten. Wie diese Professionalisierung von Lehramtsstudierenden in Forschung und Lehre umgesetzt werden kann, wird exemplarisch an einem Projektseminar zum inklusiven naturwissenschaftlichen Unterricht vorgestellt. Das BMBF Projekt »Nawi-In «1 vereint Forschung und Lehre, indem es die Kompetenzentwicklung Studierender im Projektseminar beforscht. Dies wird durch videobasierte Kompetenzforschung begleitet (Riegel 2013).

Schlüsselwörter: Forschendes Lernen, Inklusion, inklusiver Naturwissenschaftsunterricht, Lehrer*innenbildung, videobasierte Kompetenzforschung

\section{Summary}

Professionalization of student teachers for inclusive science teaching

Germany ratified the UN Convention on the Rights of Persons with Disabilities (United Nations 2006, Article 24), and is therefore bound by contract to make »Education for All « possible (UNESCO 2015) in the German school system. Now there are two challenges for science education: first, the school education is divided in different subjects with different 
subject contents. The contents have to be prepared inclusively and subject-related. Second, the complexity of the subject contents rises noticeably from primary to secondary school, so it gets more difficult to regard all needs of a heterogeneous group of students adequately (Musenberg and Riegert 2015, 5). Therefore, a professionalization of teacher students should target these challenges to prepare them effectively for their working field. How the implementation of teacher students' professionalization can be orchestrated is shown exemplary by a project seminar designed for inclusive science teaching. Research and teaching is united through the $\gg$ Nawi-In «-project - funded by the Federal Ministry of Education and Research - by investigating the students' development of competencies. This development is monitored by video-based competency research (Riegel 2013).

Key words: inclusion, inclusive science education, inquiry-based learning, teacher education, video-based competency research

\section{$1 \quad$ Einleitung}

Mit der Ratifizierung der Convention on the Rights of Persons with Disabilities (CRPD) der Vereinten Nationen, in der Menschen mit Behinderung die Teilhabe an einer umfassenden Bildung zugesichert wird (United Nations 2006, Artikel 24), verpflichtete sich die deutsche Bundesregierung im Jahr 2009 den Forderungen der Konvention und somit auch den Inhalten des oben genannten Artikels nachzukommen (UNESCO 2009, 15). Zehn Jahre nach der Ratifizierung ist inklusiver Unterricht noch nicht in allen Schulen angekommen und insbesondere die Ausbildung angehender Lehrkräfte wurde nur vereinzelt an die Forderungen der UN-Behindertenrechtskonvention angepasst (Schönig und Fuchs 2016, 24-25).

Aufgrund der langen sonderpädagogischen Tradition sowie der Integrationsbewegung in den 1970er Jahren - und bekräftigt in der Verwendung des Begriffs in der Konvention - wird der Begriff > Inklusion < häufig alleinig auf die Differenzlinie der Behinderung bzw. des sonderpädagogischen Förderbedarfs fokussiert (Wocken 2014). Im Gegensatz zu diesem engen Inklusionsbegriff steht ein weites Verständnis von Inklusion (Werning 2015, 16-17). Nach dem weiten Verständnis geht es um die Reduzierung von Exklusion und jeglicher Diskriminierung sowie den Abbau von physischen und mentalen Barrieren. Es wird die Partizipation aller Menschen an Kultur, Bildung und Gesellschaft angestrebt (UNESCO 2009). Für Lehrkräfte bedeutet dies, sich darauf zu konzentrieren, welche Ressourcen und Bereicherungen Schüler*innen durch ihre Potenziale in den Unterricht einbringen (z.B. Fähigkeiten und Fertigkeiten, Wissen, Interesse etc.), um eine Lernumgebung zu schaffen, die alle Lernenden unterstützt und ihnen hilft, ihre Potenziale zu erweitern (Abels 2019; Gudjonsdottir und Óskarsdóttir 
2016, 6). Die Anerkennung von Vielfalt in der Schule bedeutet weder jede*n gleich, noch alle unterschiedlich zu unterrichten. Es gilt, die verschiedenen Zugänge der Schüler*innen zu geteilter Erfahrung zu verstehen und bereitzustellen (Ainscow et al. 2006, 295-308).

Da Heterogenität in Schulklassen Realität ist, die sich im Unterrichtsalltag von praktizierenden Lehrkräften häufig auch als Überforderung zeigt, sollten bereits angehende Lehrkräfte in Hochschulen ausreichend auf diesen Alltag vorbereitet werden (Amrhein und Reich 2014, 32-33). Das Spannungsfeld eines engen und weiten Verständnisses von Inklusion muss auch in der Ausbildung zukünftiger Lehrpersonen diskutiert werden, die sich zwischen Illusion und Vision von Inklusion bewegen können müssen. Die Besonderheit bei der Ausbildung angehender Lehrkräfte ist, dass das deutsche Schulsystem in einzelne Fächer aufgegliedert ist und die universitäre Ausbildung demnach ebenso stattfindet. Neben dem Studium erziehungs- bzw. bildungswissenschaftlicher Inhalte sind für Regelschullehrämter die gewählten Fächer zentral, in denen neben den fachwissenschaftlichen Grundlagen fachdidaktische Inhalte vermittelt werden. Die Studierenden professionalisieren sich darüber hinaus in Unterrichtspraktika speziell auf ihre Fächer bezogen, jedoch selten auf die notwendige Entwicklung hin, ihren Fachunterricht in stark heterogenen Schulklassen durchzuführen (Troll et al. 2019, 537-538). Ein Grund dafür ist der fehlende Transfer von inklusiver Pädagogik auf die Unterrichtsfächer, ein anderer die fehlende Weiterbildung von Dozierenden an den Hochschulen. Diese müssen sich der Herausforderung stellen, das Thema >Inklusive Bildung< in ihre fachdidaktischen Lehrveranstaltungen zu integrieren und mit den Unterrichtsfächern zu verknüpfen (Abels und Schütz 2016). Zusätzlich zu dieser Lücke im Studium der Fachdidaktiken finden kaum Fort- und Weiterbildungen für Lehrkräfte statt, die sich bereits im Schuldienst befinden (Weishaupt 2015). Damit Inklusion besser Eingang in die Schulen findet, sollten Lehrkräfte in ihrer Berufspraxis mehr unterstützt und angehende Lehrkräfte gezielter auf die Umsetzung inklusiven Fachunterrichts vorbereitet werden.

Im vorliegenden Beitrag wird die Umsetzung von Inklusion in der Naturwissenschaftsdidaktik fokussiert. Die Professionalisierung angehender Lehrkräfte ist eine notwendige Bedingung für die gelingende Umsetzung von Inklusion im Unterrichtsalltag und so auch im Fachunterricht. Die angehenden Fachlehrpersonen tragen die Inhalte ihres Studiums in die schulische Praxis und gestalten den Unterricht für künftige Generationen von Schüler*innen. Für eine gelingende und progressive Umsetzung von Inklusion in den Schulen sollten sich also Hochschulen der Herausforderung in Forschung und Lehre stellen, Lehramtsstudierende auf das Unterrichten inklusiv gestalteter Fachinhalte vorzubereiten (Abels und Schütz 2016, 425).

An dieser Leerstelle knüpft das vom BMBF geförderte Projekt Nawi-In - Naturwissenschaftlichen Unterricht inklusiv gestalten an. Im Projekt werden die Professionalisie- 
rung und der Kompetenzerwerb angehender Lehrkräfte beforscht und gefördert. Das Ziel des Forschungsprojekts ist, eine Antwort auf die übergeordnete Fragestellung zu finden, welche professionelle Kompetenzentwicklung für inklusiven naturwissenschaftlichen Unterricht sich bei Lehramtsstudierenden im Masterstudium feststellen lässt. Ort der Forschung ist das Projektband, das ausführlich unter Abschnitt 3.2 beschrieben wird. Dabei werden zwei Schulstufen einbezogen und verglichen: Sachunterricht mit den Bezugsfächern Biologie, Chemie und Physik in der Primarstufe sowie Biologie und/oder Chemie in der Sekundarstufe I.

Ziel des vorliegenden Beitrages ist die Wichtigkeit von Inklusion in der Professionalisierung angehender Lehrkräfte herauszustellen und die Professionalisierung exemplarisch in der Naturwissenschaftsdidaktik zu erläutern. Es wird aufgezeigt, welche Rolle der Fachdidaktik in der Ausbildung angehender Lehrkräfte zukommt. Um eine Überforderung in der späteren Berufspraxis zu vermeiden, sollen angehende Lehrkräfte entsprechend auf den Umgang mit einer heterogenen Schüler*innenschaft vorbereitet werden. »Bildung für alle« (UNESCO 2015) kann nur umgesetzt werden, wenn in der Ausbildung der Lehrkräfte Inklusion auch in der Fachdidaktik konsequent thematisiert und als fester Bestandteil in die fachdidaktische Hochschullehre integriert wird.

\section{Professionalisierung für die Umsetzung von Inklusion}

Die European Agency for Development in Special Needs Education (2012) hat in dem europaweiten Projekt »Teacher Education for Inclusion « untersucht, wie die Lehrer*innenbildung in Hinblick auf einen inklusiven Unterricht ausgerichtet werden kann. Ausgehend von der Annahme, dass die Lehrer*innenbildung einen wesentlichen Ansatz für die Entwicklung einer inklusiven Bildung darstellt, werden nicht nur zentrale Voraussetzungen der Lehrkräfte formuliert, sondern auch Empfehlungen für die Gestaltung der Lehrer*innenbildung ausgesprochen. »Lehramtsstudierende brauchen Erfahrungen aus erster Hand in der Arbeit mit Lernenden mit unterschiedlichen Bedürfnissen und Lehrende, die für die Arbeit in inklusiven Settings ausgebildet sind. Lehramtsstudierende müssen in Praktika die Umsetzung des Erlernten ausprobieren können und die Gelegenheiten zu Praktika in inklusiven Settings erhalten « (European Agency for Development in Special Needs Education 2012, 24-25).

Die European Agency legt den Fokus aber nicht nur auf die direkten Erfahrungen, die Studierende in der Praxis mit Diversität erlangen, sondern vor allem auf die explizite Reflexion und das konstruktive Feedback, entlang dessen die Studierenden ihre Kompetenzen weiterentwickeln können. Für die Gestaltung von Qualifizierungsmaßnahmen wird ein Ausgleich von Theorie-, Reflexions- und Praxisphasen gefordert (WiFF 2013). Die bisherige Forschung im Professionalisierungskontext zeigt, dass die Notwendig- 
keit der theoriegeleiteten und reflektierten Auseinandersetzung mit und in der Praxis besteht, um Kompetenzaufbau zu ermöglichen (Loucks-Horsley et al. 2003). Für einen nachhaltigen Kompetenzaufbau in der Lehrer*innenbildung ist es entscheidend, dass die entsprechenden Hochschullehrenden die Verantwortung für eine inklusionsorientierte Ausbildung angehender Lehrkräfte übernehmen. Dafür müssen zunächst bisherige Hochschulcurricula an die Anforderungen der unterrichtlichen Wirklichkeit angepasst und zukunftsorientiert verändert werden (vgl. Abschnitt 2.2). Diese formale Anpassung kann jedoch nur wirksam werden, wenn der Fokus auf die zu entwickelnden Kompetenzen angehender Lehrkräfte gelegt wird und somit eine Veränderung in der Lehre tatsächlich stattfindet.

\subsection{Kompetenzen von Lehrkräften für einen inklusiven Fachunterricht}

Zunächst stellt sich die Frage, was unter inklusivem Unterricht zu verstehen ist. Im Beschluss der Kultusministerkonferenz zur inklusiven Bildung (2011) wird die Gestaltung inklusiven Schulunterrichts so dargestellt, dass er sowohl den >Standards und Zielsetzungen für allgemeine schulische Abschlüsse < entsprechen als auch die individuellen Kompetenzen der Lernenden berücksichtigen sollte (ebd., 9). Maßnahmen dafür sind zum Beispiel innere und äußere Differenzierung, die Nutzung von Unterrichtsmedien und technischen Gestaltungsmöglichkeiten für gehandicapte Schüler*innen, die Gestaltung in leichter Sprache, inklusiv gestaltete Lehr- und Lernmaterialien, kreative Entfaltungsmöglichkeiten in der Lernumgebung und eine lernprozessbegleitende Diagnostik (ebd., 9-10). Aus diesen Vorgaben heraus werden Anforderungen an die Kompetenzen von Lehrkräften gestellt, die sich im Spannungsfeld zwischen den zu erreichenden Standards und den individuellen Lernprofilen der heterogenen Schüler*innenschaft ergeben. Moser und Kropp (2014) haben im Rahmen des Forschungsprojekts KIS (Kompetenzen in inklusiven Settings) ein Kompetenzcluster erstellt, das folgende (Handlungs-)Kompetenzen gemäß den Anforderungen in der Praxis von den (sonderpädagogischen) Lehrkräften erfordert: Beratungs- und Organisationskompetenz, binnendifferenzierte Unterrichtung, Lern- und Entwicklungsförderung, behinderungsspezifische Kommunikation, interdisziplinäre Kooperation, Förderung des sozialen Lebens sowie Lernstands- und Entwicklungsdiagnostik (ebd., 5). Doch nicht nur an Sonderpädagog*innen werden diese Anforderungen gestellt. Für die Umsetzung von Inklusion an Schulen müssen auch Regelschullehrkräfte zu sogenannten »spezialisierten Generalisten « (Sawalies, Veber, Rott und Fischer 2013, 5) ausgebildet werden.

Wird von diesen Anforderungen an Lehrkräfte ausgegangen, müssen in einem nächsten Schritt die Inhalte der universitären Lehre und damit auch Hochschulcur- 
ricula so modifiziert werden, dass die Entwicklung professioneller Kompetenzen zur Erfüllung der Anforderungen unterstützt werden kann. Bezogen auf inklusiven Fachunterricht bestehen einige Herausforderungen, für die die Entwicklung entsprechender Kompetenzen eine zentrale Aufgabe darstellt. Bisher gibt es jedoch noch kein Modell professioneller Kompetenz für inklusiven Fachunterricht. Allgemeine Modelle zur Professionalisierungskompetenz müssen auf ihre Eignung als Grundlage geprüft und ggf. erweitert werden.

Im Projekt Nawi-In (vgl. Abschnitt 1) wurden bereits Prädiktoren für einen gelingenden inklusiven naturwissenschaftlichen Unterricht identifiziert. Diese Prädiktoren wurden anhand eines Systematic Literature Review (Fink 2009) ermittelt, indem Suchbegriffe zu inklusivem naturwissenschaftlichen Unterricht in drei Datenbanken (ERIC, FIS Bildung und Scopus) recherchiert wurden (Brauns et al. in Vorb.). Nach einem Titel- und einem anschließenden Abstractscreening wurde die Anzahl von 10.787 Artikel auf eine Stichprobe von 286 Artikeln gefiltert und aus dieser mittels qualitativer Inhaltsanalyse (Kuckartz 2016) induktive Kategorien gebildet. Mit den vorliegenden Prädiktoren sollen die Studierenden im Projektband (s. Abschnitt 3.2) inklusiven naturwissenschaftlichen Unterricht planen, durchführen und reflektieren (s. Abb. 1).

In der fachdidaktischen Ausbildung sollte der Fokus darauf liegen, Fachwissen verständlich aufbereiten und Grundlagenwissen bei den Schüler*innen ausbilden zu können (Amrhein und Reich 2014, 33). Lerninhalte sollten auf einem gemeinsamen Tätigsein und Erleben gründen und daraus ein individualisierter Lernprozess abgeleitet werden (Ziemen 2014, 53). Angehende Lehrkräfte müssen sich mit fachspezifischen Aneignungsmodi, vielfältigen Zugängen zu Lerngegenständen und Schüler*innenvorstellungen auseinandersetzen, um Lernbarrieren, die das Fach stellt, zu erkennen und zu beseitigen (Abels und Schütz 2016). Naturwissenschaftsdidaktische Publikationen hierzu beziehen sich in der Regel auf einzelne Diversitätsdimensionen, wie etwa Geschlecht, Migration oder sonderpädagogischer Förderbedarf (Markic und Abels 2014, 273). Publikationen im Bereich des inklusiven naturwissenschaftlichen Unterrichts, die sich an einem weiten Inklusionsbegriffs orientieren, sind selten und dann häufig noch theoretisch bzw. normativ formuliert. Sie tendieren zu allgemein-pädagogischen statt fachdidaktischen Empfehlungen (Stroh 2015).

Im DFG geförderten »Netzwerk inklusiver naturwissenschaftlicher Unterricht « (NinU) wurde bisher ein Konsens erarbeitet, dass nach dem weiten Inklusionsverständnis inklusiver naturwissenschaftlicher Unterricht » allen Lernenden - unter Wertschätzung ihrer Diversität und ihrer jeweiligen Lernvoraussetzungen - die Partizipation an individualisierten und gemeinschaftlichen fachspezifischen Lehr-Lern-Prozessen zur Entwicklung einer naturwissenschaftlichen Grundbildung ermöglicht « (Menthe et al. 2017, 801). Im naturwissenschaftlichen Unterricht zeigen sich bei der Umsetzung aber 
große Herausforderungen, zum Beispiel in der Gestaltung der fachlichen Lernumgebung (Abels 2016), der Fachsprache (Abels und Markic 2013; Riebling 2013), der Schüler*innenvorstellungen (Menthe und Hoffmann 2015) und der naturwissenschaftlichen Arbeitsweisen (Andersson und Gullberg 2014).

Diesen Herausforderungen muss sich die fachdidaktische Lehrer*innenbildung widmen und die dargestellten Aspekte müssen auch offiziell Eingang in die Hochschulcurricula finden, um langfristig Teil der Ausbildung von Lehrpersonen zu werden.

\subsection{Curricula Inklusiver Lehrer*innenbildung}

Exemplarisch werden Angebote verschiedener Hochschulen zu Inklusiver Bildung kurz vorgestellt. Dies soll die Aufbruchsstimmung, die bezüglich Inklusiver Bildung herrscht, hervorheben, nicht den derzeitigen Stand der Umsetzung abbilden, der sich vermutlich durch die Qualitätsoffensive Lehrerbildung in Veränderung befindet. Die genannten Universitäten zeichnen sich dadurch aus, dass durch curriculare Änderungen in der Hochschullehre sowohl ein stärkerer Praxisbezug für die Lehramtsstudierenden erwirkt wurde als auch eine nachhaltige Verzahnung von Regelschul- und Sonderpädagogik. Dies ist als kurze Übersicht zu verstehen, die Veränderungen in der Lehrer*innenbildung hin zu einer inklusiveren und praxisbezogeneren Ausbildung auf Hochschulebene darstellt. Es wird kein Anspruch auf Vollständigkeit erhoben, sondern auf hochschuldidaktische Publikationen zu diesem Thema zurückgegriffen, worüber sich die folgende exemplarische Auswahl der Universitäten erklärt.

An der Carl-von-Ossietzky-Universität in Oldenburg werden Qualifikationen in drei Modulen erworben, in denen stark auf die theoretische Fundierung und Reflexion der praktischen Erfahrung gesetzt wird: Biografische Erfahrung statt Belehrung, differenzierende Förderkompetenzen erwerben und Forschendes Lernen (Fichten et al. 2006, 133-151). Im ersten Modul sollen die Teilnehmenden ein Verständnis individueller Lernwege erlangen. Im zweiten Modul liegt der Schwerpunkt auf Diagnostik sowie der Planung, Durchführung und Reflexion von Unterricht für heterogene Lerngruppen. In letzterem Modul beforschen die Studierenden gemeinsam mit Lehrkräften die eigene Praxis mit dem Ziel einer kritisch-reflexiven Haltung gegenüber dem Arbeiten mit heterogenen Schulklassen (Obolenski 2004).

An der Universität Bremen absolvieren Studierende aller Lehrämter eine Basisqualifizierung von 15 Leistungspunkten (450 Zeitstunden) mit den Schwerpunkten Interkulturelle Bildung, Inklusive Pädagogik und Deutsch als Zweitsprache. Sonderschullehramt kann ausschließlich in Kombination mit dem Grundschullehramt studiert werden. Für die Sekundarstufe wird ein sonderpädagogischer Weiterbildungsstudiengang etabliert (Seitz 2011, 50-54). 
An der Pädagogischen Hochschule Heidelberg wurde 2011 unter der damaligen Leitung von Rektorin Prof. Anneliese Wellensiek ein Übergreifender Studienbereich (ÜSB) für alle Lehramtsstudierenden (Primar- und Sekundarstufe I) eingerichtet. Eins von drei Modulen im Umfang von fünf bis sieben Leistungspunkten fokussiert dabei auf den Bereich Inklusion und Diversität.

»Studierende sollen sich in diesem Modul für ethische, rechtliche, pädagogische und didaktische Aspekte inklusiver Bildung öffnen. Sie sollen als angehende Lehrer_Innen lernen, Diversität [...] als Chance für gemeinsame Lern- und Entwicklungsprozesse wahrzunehmen und entsprechende Rahmenbedingungen für Lehr- und Lernsituationen zu schaffen « (Pädagogische Hochschule Heidelberg 2019).

Auch in Österreich, das ein in Ansätzen vergleichbares Bildungssystem aufweist, wurden Curricula umgearbeitet. An der Pädagogischen Hochschule Oberösterreich ist das Fach Inklusive Pädagogik im Curriculum verankert und von allen Lehramtsstudierenden zu belegen (vier SWS Pflicht und weitere vier SWS Wahlmöglichkeit; Feyerer 2004, 339-349). Der Schwerpunkt »Förderung individueller Begabungen, umfassende Partizipation an Bildung, inklusive Pädagogik « ist darüber hinaus in viele Module des Curriculums verflochten.

An der Universität Wien ist das Pflichtmodul »Inklusive Schule und Vielfalt « (fünf ECTS-Punkte) in den Bildungswissenschaften zu belegen. Manche Fachdidaktiken haben zusätzlich eigene Module mit dem Fokus auf Diversität entwickelt (Abels und Schütz 2016).

An der Leuphana Universität Lüneburg ist Inklusion in der Lehrer*innenbildung im Bachelorstudiengang Lehren und Lernen im Professionalisierungsbereich verankert. Festgeschrieben ist das Grundlagenseminar (fünftes Semester) und das Aufbauseminar (sechstes Semester) »Inklusion - Chancen und Herausforderungen I und II « für die Regelschule im Curriculum der Universität (ZZL 2018).

Insgesamt scheint inklusive Pädagogik als Querschnittsthematik an einigen Hochschulen zunehmend mitgedacht zu werden. Ein umfassender und systematischer Blick auf deutschsprachige Hochschulen würde sich lohnen, kann aber im Rahmen dieses Artikels nicht geleistet werden. Die kurze Übersicht zeigt, dass ein Desiderat nach wie vor die fachdidaktische Ausbildung für Inklusion zu sein scheint. Die fachdidaktische Lehrer*innenbildung ist noch nicht oder nur vereinzelt bzw. unsystematisch auf das Unterrichten heterogener Schüler*innengruppen ausgerichtet und bietet den Studierenden damit eher Orientierung im exklusiven Paradigma (Amrhein und Reich 2014).

Bezogen auf Inklusion und Fachdidaktik fand 2018 im ministerial geförderten Projekt »Inklusive Basiskompetenzen in der Lehrerinnen- und Lehrerbildung « (IBaLL) eine Ringvorlesung mit dem Titel »Das inklusive Klassenzimmer « an der Leuphana 
Universität Lüneburg statt, in der Expert*innen verschiedener Fachdisziplinen (Sport, Sachunterricht, Mathematik und Sprachunterricht) die Studierenden für die Umsetzung von Inklusion in den unterschiedlichen Schulfächern sensibilisierten. In einem anschließendem »Denkraum « tauschten sich Lehrende der Universitäten mit den Expert*innen aus, um die Lehrer*innenbildung an der Leuphana Universität weiterzuentwickeln und Kooperationen anzubahnen, sodass den Studierenden Kompetenzentwicklung für inklusiven Unterricht sukzessive ermöglicht wird.

Neben Veränderungen in den Hochschulcurricula nimmt die Forschung für und mit der Praxis in der Lehrer*innenbildung eine wichtige Position ein, um festzustellen, ob die Veränderungen der Curricula auch tatsächlich in der Lehre umgesetzt werden. Besonders hat sich in der Beforschung von Kompetenzen (angehender) Lehrkräfte die videobasierte Forschung etabliert.

\subsection{Videobasierte Kompetenzforschung in der Lehrer*innenbildung}

Die Videoforschung ist in der empirischen Bildungsforschung, speziell in der Unterrichtsforschung und in der Lehrer*innenbildung zu einem wichtigen Instrumentarium geworden. In einigen fachdidaktischen Disziplinen werden und wurden videobasierte Forschungsprojekte durchgeführt (Riegel 2013, 18). Besonders verbreitet scheint Videoforschung im Mathematik-, Deutsch- und naturwissenschaftlichem Unterricht sowie im fremdsprachlichen Unterricht zu sein (ebd., 19). Im Bereich der Mathematik- und Naturwissenschaftsdidaktik sind vor allem die TIMSS-Studie (Trends in International Mathematics and Science Study) (Wendt et al. 2016), die IPN-Videostudie (Leibniz-Institut für die Pädagogik der Naturwissenschaften und Mathematik, Kiel) (Seidel et al. 2005) und das ViU-Projekt (Videobasierte Unterrichtsanalyse: Early Science - Theoretische Modellierung und empirische Erfassung der Kompetenzen zur Analyse der Lernwirksamkeit von naturwissenschaftlichem Grundschulunterricht) (WWU Münster 2019) zu nennen. Diese Videostudien liefern durch ihre Ergebnisse auch wichtige Beiträge zur Kompetenzforschung von Lehrkräften. Durch videobasierte Kompetenzforschung kann das Unterrichtshandeln einer Lehrkraft sichtbar gemacht werden, um im Anschluss die Reflexion ihres Handelns zu unterstützen und somit eine Weiterentwicklung und Erweiterung des Handlungsrepertoires zu fördern. Studien zeigen in Hinblick auf den Einsatz von videobasierten Ansätzen in der Lehrer*innenbildung, dass diese sowohl zur Steigerung der subjektiven Kompetenzeinschätzung führen (Gröschner et al. 2018, 60), als auch lernförderlich und motivierend auf Studierende wirken können (Hoeks 2011, 56). Es wird davon ausgegangen, dass die Arbeit mit Unterrichtsvideos angehenden Lehrkräften dabei hilft, einen professionellen Habitus zu entwickeln und unterrichtsrelevante Kompetenzen zu verbessern (Sherin 2007, 
383-396). Dies kommt durch die Schulung der Unterrichtswahrnehmung zustande, die als professional vision bezeichnet wird. Anders als bei Goodwin (1994), der bei professional vision davon ausgeht, dass eine professionsspezifische Tätigkeit zur Schulung der Wahrnehmung führt, verstehen van Es und Sherin (2008) diesen Begriff als professionellen Blick der Lehrkraft auf das Unterrichtshandeln. Dabei sollen wichtige von unwichtigen Informationen getrennt werden (selective attention, noticing) und aus den Wahrnehmungen der Videoinhalte auf Basis des professionellen Wissens Schlussfolgerungen gezogen werden (knowledge-based reasoning). Das gezeigte Handeln in den Unterrichtsvideos kann bewertet und/oder interpretiert werden (Lindmeier 2013, 49). Besonders die Interpretation wird von van Es und Sherin (2008) als wichtig erachtet, da diese sinnkonstituierend ist und die Reflexion der gezeigten Situationen ermöglicht.

Förderlich sind sowohl Fremdreflexionen als auch die Reflexion eigener Praxis. Letzteres heißt, dass (angehende) Lehrkräfte im Sinne einer video-based intervention das geschulte professionelle Handeln in der Praxis erproben und die Videos von ihrem eigenen Unterricht mittels professioneller Wahrnehmung analysieren, ihr Handeln reflektieren und weiterentwickeln (Janik et al. 2013). Die Reflexion wird als » eine Vorbedingung professionellen Handelns « gesehen (ebd., 64). Dadurch entsteht ein Zyklus aus Theorie und Praxis, der sich positiv und nachhaltig auf die professionelle Kompetenzentwicklung der Lehramtsstudierenden auswirkt (Leonhard und Abels 2017). Seidel et al. (2011) konnten feststellen, dass Lehrkräfte, die ihren eigenen Unterricht reflektieren, mehr Aspekte wahrnehmen (noticing) als Lehrkräfte, die Unterricht anderer Personen analysieren. Dafür waren die Eigenreflexionen weniger kritisch als die Fremdreflexionen und es wurden weniger Handlungsalternativen generiert. Abels (2019) konnte zeigen, dass sowohl in Eigen- als auch in Fremdreflexionen kaum Aspekte inklusiven Lernens zur Sprache kommen, insbesondere keine fachdidaktischen Aspekte inklusiven Lernens. Hierfür benötigen Lehrpersonen ein hohes Maß an Strukturierung und Unterstützung, um diese Aspekte der Reflexion zugänglich zu machen.

Der Effekt der Wahrnehmung und Reflexion durch das Zeigen und unterstützte Interpretieren von Unterrichtsvideos wird im vorliegenden Forschungsprojekt genutzt.

\section{Inklusion als Aufgabe für die Naturwissenschaftsdidaktik}

Die Umsetzung von Inklusion im Fachunterricht ist eine fachdidaktische Aufgabenstellung. Um Inklusion und Fachdidaktik gemeinsam zu denken, müsste letztere breiter aufgestellt und nicht nur von der Perspektive der Fachinhalte aus gedacht werden, 
sondern von der Perspektive des lernenden Individuums heraus (Stroh 2015). Dies gestaltet sich besonders in Deutschland schwierig. Die Ausbildung in den jeweiligen Fächern orientiert sich am Fachwissen und den fachwissenschaftlichen Methoden und im Vergleich zu anderen Ländern weniger an der didaktischen Ausgestaltung - noch weniger an der didaktischen Ausgestaltung eines inklusiven Fachunterrichts. Befunde zeigen, dass die Ansprüche an Fach- und an inklusiven Unterricht schwer zu vereinen sind, insbesondere in der Sekundarstufe (Abels, Heidinger, Koliander und Plotz, 2018; Musenberg und Riegert 2015). Die These ist, dass ein zunehmender fachlicher Anspruch Exklusionsmechanismen forciert, da die Komplexität der Unterrichtsinhalte und die damit verbundenen Anforderungen an die Schüler*innen zunehmen (ebd.).

Auch die Anforderungen an die Lehrkräfte steigen, da diese sich ständig in einem Spannungsfeld von Orientierung an fachlichen Inhalten und einer inklusiven Unterrichtsgestaltung befinden (Abels 2016). Um auch die angehenden Lehrkräfte zu entlasten und einer Überforderung im Bereich der inklusiven Unterrichtsgestaltung und -durchführung entgegenzuwirken, ist die Entwicklung fachdidaktischer Module für die konsequente Vorbereitung auf inklusiven Fachunterricht gefordert. Bestehende fachdidaktische Ansätze müssen auf ihre Eignung für den Umgang mit Vielfalt geprüft werden. Die Ansätze müssen für spezifische fachliche Konzepte und Kontexte modelliert werden (Markic und Abels 2014).

\subsection{Professionalisierung für inklusiven naturwissenschaftlichen Unterricht}

Um die Professionalisierung angehender Lehrkräfte inklusiv entlang fachdidaktischer Prinzipien gestalten zu können, müssen Lehr-/Lernformate geprüft und auf ihre Umsetzbarkeit getestet werden.

Stroh $(2015,114)$ prüft Ansätze, die in einem Spannungsfeld zwischen Fach und Subjekt beide Pole vereinen könnten. Innerhalb dieses Spannungsfeldes verortet Stroh inklusiven Fachunterricht. Ist Unterricht auf die Entfaltung des Individuums ausgelegt, bewegt sich die primäre Orientierung der Fachdidaktik von der Sachorientierung zur Subjektorientierung (ebd.). Eine Orientierung zur Sache hin würde dazu führen, dass das zu vermittelnde Wissen exponentiell anstiege und die Ziele des inklusiven naturwissenschaftlichen Unterrichts noch schwerer zu erreichen wären (ebd., 115). Allerdings dürften die Ansprüche des Faches nicht zugunsten einer Subjektorientierung vernachlässigt werden (ebd., 113). Stroh hat Unterrichtsmethoden nach dem Conceptual Change Ansatz (Kahlert 2009) und das forschend-entwickelnde Unterrichtsverfahren nach Schmidkunz und Lindemann (2003) daraufhin geprüft, ob sie der Sache oder dem Subjekt gerecht werden. Während Conceptual Change Ansätze sich eher an den Sub- 
jekten orientierten, aber eine beständige Diagnostik und Differenzierung benötigten, die Lehrpersonen in ihrem Unterrichtsalltag kaum leisten können, orientiere sich das forschend-entwickelnde Unterrichtsverfahren in lehrerzentrierter Weise an der Sache und eigne sich nicht für inklusiven Fachunterricht.

Feyerer (2004) nennt als inklusive und potenzialorientierte Ansätze die Projektund Werkstattarbeit, Stationslernen und Freiarbeit, die als schüler*innen- und kompetenzorientierte Ansätze das individuelle Lernen der Schüler*innen in sozialer Eingebundenheit ermöglichen. Im Hinblick auf inklusiven Unterricht lassen sich diese Ansätze adaptiv gestalten, damit Schüler*innen ihr Potenzial entfalten können (Abels 2019).

Ein weiterer Ansatz ist die Idee des Auflösens traditioneller Schulfächer und das Einsetzen von Themenfeldern (Booth 2014, 63). Themen wie »Water «, »Life on earth « oder »Sources of energy« (Booth 2014, 63) lassen sich in allen drei naturwissenschaftlichen Fächern verankern (Biologie, Chemie und Physik). In diesem Zusammenhang weist Abels (2019) darauf hin, dass die Auflösung der Fächer jedoch aufgrund des aktuellen Schulsystems in absehbarer Zeit nicht geschehen wird. Realistischer ist ein Unterricht in der Fächerstruktur entlang von Kontexten (Gilbert 2006), die zum Beispiel entlang des Forschenden Lernens in unterschiedlichen Offenheitsgraden bearbeitet und darüber alle Kompetenzbereiche einbezogen werden können (Bewertung, Erkenntnisgewinnung, Fachwissen und Kommunikation) (Abels, 2019). In einem offenen Format, zum Beispiel im Rahmen einer Lernwerkstatt können Schüler*innen eigene Forschungsfragen entwickeln und anhand verschiedener Materialien selbst beantworten (Abels und Minnerop-Haeler 2015). Individuelle Lernwege werden somit von den Schüler*innen selbst gestaltet und die Leistungen von der Lehrkraft wertgeschätzt.

Insbesondere die Forschungsgruppe um Thomas Scruggs zeigt in ihren Studien, dass sich für den inklusiven naturwissenschaftlichen Unterricht das Forschende Lernen aufgrund der starken Subjekt- und Handlungsorientierung sowie der variablen Strukturierungsmöglichkeiten bewährt (z. B. Brigham, Scruggs und Mastropieri 2011). Lehrkräfte sollten nicht als reine Wissensvermittler*innen auftreten, sondern als Lernbegleitung, die den selbstbestimmten Lernprozess individuell beratend und organisierend konstruktiv unterstützen sollte (Ziemen 2014, 46). Meta-Studien zeigen den positiven Effekt des begleiteten Forschenden Lernens insbesondere für Schüler*innen mit sonderpädagogischem Förderbedarf (Therrien et al. 2011; Villanueva et al. 2012).

Das Lehr-/Lernformat des Forschenden Lernens eignet sich auch für die universitäre Ausbildung von angehenden Lehrkräften, die so in Form eines didaktischen Doppeldeckers an die Gestaltung von inklusivem naturwissenschaftlichen Unterricht herangeführt werden können (Brauns et al., in Vorbereitung). Dies ist so zu verste- 
hen, dass das Forschende Lernen auf zwei Ebenen Anwendung finden kann: zum einen als Lernform im naturwissenschaftlichen Unterricht, die Lehramtsstudierende erproben können, und zum anderen als hochschuldidaktischer Ansatz, entlang dessen die Studierenden eigene Forschungsprojekte planen und gestalten. Das Ziel ist, dass die Studierenden einen forschenden Habitus entwickeln, der sie als angehende Lehrpersonen beständig Unterricht hinterfragen und adaptieren lässt (Huber 2006). Im nachfolgenden Abschnitt wird die Umsetzung dieser Ansätze im Rahmen des Nawi-In Projekts der Leuphana Universität Lüneburg genauer beschrieben.

\subsection{Das Projektband zum Forschenden Lernen in den Naturwissenschaften}

Neben den bildungswissenschaftlichen Seminaren integrieren auch die Fachdidaktiken das Thema >Inklusion< in ihre Module, allerdings bisher nicht systematisch und abhängig von den Kompetenzen der jeweiligen Dozierenden (vgl. Abschnitt 2.2). Im Rahmen der Neustrukturierung der Masterstudiengänge im Grund-, Haupt- und Realschulstudium (Masterabschluss mit 300 Leistungspunkten) in Niedersachsen wurden die Wissenschaftsorientierung und das Forschende Lernen als zentrale Elemente aufgenommen (Niedersächsisches Kultusministerium o.J.). Diese Elemente werden im Projektband umgesetzt, welches für alle Lehramtsstudierenden verpflichtend ist. Das Projektband kann in einem der beiden studierten Fächer oder dem Professionalisierungsbereich Bildung belegt werden. Das Modul findet in Form eines Projektseminars vom ersten bis zum dritten Mastersemester statt und liegt dabei auch parallel zur halbjährigen Praxisphase in der Schule, die von den Studierenden im zweiten Mastersemester absolviert werden muss. Die Studierenden sind Praktikumsschulen zugeordnet und werden dort von Mentor*innen bei der Planung, Durchführung und Reflexion von Unterricht betreut. Eingebettet ist die Praxisphase in ein Vorbereitungs-, Begleit- und Nachbereitungsseminar. Fragestellungen, die sich in der Schulpraxis ergeben, sollen anhand wissenschaftlicher Methoden von den Studierenden untersucht werden, damit diese eine forschende Haltung für ihr späteres Berufsfeld einnehmen können (ebd.). Ziel jedes Projektbands ist, dass die Studierenden eigene Forschungsprojekte initiieren und nach wissenschaftlichen Kriterien designen, durchführen und präsentieren. Angelehnt ist die Konzeption des Projektbands an die Oldenburger Teamforschung, die zum Ziel hat, dass Studierende im Rahmen einer selbst gewählten Studie im Praxissemester ihr späteres Berufsfeld erforschen und zu ihrer eigenen Professionalisierung beitragen (vgl. Abschnitt 2.1 und 2.2). Zusätzlich zu den neuen Erkenntnissen der Studierenden für ihre eigene Entwicklung tragen deren Forschungsarbeiten zur Weiterentwicklung in Schulen und universitärer Lehre bei (Fichten 2006, 135). 


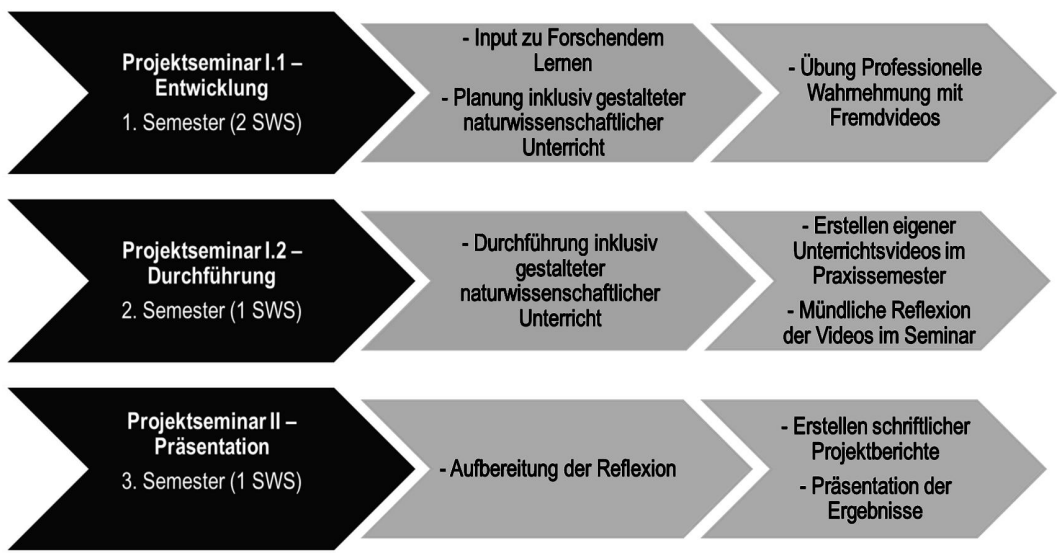

Abbildung 1: Konzeption Projektband »Forschendes Lernen im inklusiven Naturwissenschaftsunterricht»

Zur Kompetenzentwicklung angehender Naturwissenschaftslehrkräfte werden die beiden Projektbänder »Inklusion im naturwissenschaftlichen Unterricht in der Grundschule « für die Primarstufe im Sachunterricht mit naturwissenschaftlichem Bezugsfach und »Forschendes Lernen im inklusiven Naturwissenschaftsunterricht « für die Sekundarstufe I für die Fächer Biologie und Chemie an der Leuphana Universität Lüneburg angeboten (vgl. Abbildung 1). Die Studierenden werden im Projektband fächerübergreifend ausgebildet, das heißt, es werden fachdidaktische Ansätze gewählt, die in allen drei Naturwissenschaften gängig sind, wie zum Beispiel das Forschende Lernen. Im ersten Semester erlernen und vertiefen die Studierenden theoretische Grundlagen zu inklusiv gestaltetem Fachunterricht (Musenberg und Riegert 2015), erwerben Wissen über Definitionen (Menthe et al. 2017; UNESCO 2009), klären Begrifflichkeiten im Zusammenhang mit dem weiten und engen Inklusionsverständnis (Werning 2014) und diskutieren Prinzipien inklusiven Unterrichtens (Feyerer 2012). Es kommen Methoden wie Gruppenpuzzle, Kugellager u. Ä. zum Einsatz, bei dem die Studierenden auch aufgefordert werden, fächerverbindende wie fächerspezifische Aspekte zu thematisieren (Arbeit mit Experimenten, Modellen, Formelsprache etc.). Die Studierenden wenden den Ansatz des Forschenden Lernens aktiv an, bevor sie ihn im inklusiven naturwissenschaftlichen Unterricht einsetzen sollen, erfahren Lernbegleitungsstrategien und damit verknüpfbare Differenzierungsmaßnahmen. Zusätzlich bekommen die Studierenden Input zu fachdidaktischen Forschungsmethoden, die sie in ihrem hochschuldidaktischen Forschungsprojekt anwenden und umsetzen können. Der Schwerpunkt liegt dabei auf der Videografie und der Videoanalyse (vgl. Abschnitt 2.3). 
Anhand von Videovignetten aus der Unterrichtspraxis erfahrener Naturwissenschaftslehrkräfte schulen Studierende ihre Wahrnehmung von inklusiven Unterrichtssettings und werden dazu angeregt, sich kritisch mit dem Handeln der Lehrkraft auseinanderzusetzen und Handlungsalternativen zu entwickeln (Alsawaie und Alghazo 2010). Von dieser Vorbereitung profitieren die Studierenden in der anschließenden Praxisphase, in der sie ihren zuvor im Seminar entwickelten Fachunterricht in inklusiven Settings umsetzen können. Insgesamt werden zwei Unterrichtseinheiten von den Studierenden videografiert, in denen sie ihren geplanten inklusiven naturwissenschaftlichen Unterricht durchführen. Die erste gefilmte Unterrichtseinheit wird von den Studierenden im Projektbandseminar gemeinsam reflektiert - wie zuvor die eingesetzten Fremdvideovignetten - und ein zweites Unterrichtsvideo während einer Unterrichtseinheit erstellt, in der die Ergebnisse der Reflexion umgesetzt werden sollen. Ob dies gelungen ist, wird erneut der Reflexion zugänglich gemacht.

Im Projektband zum Forschenden Lernen ist der Einsatz der Fremd- und Eigenvideos als video-based intervention vorgesehen (vgl. Abschnitt 2.3). Die Intervention zielt auf eine Verbesserung des Lehrer*innenhandelns ab, da den Beobachtenden ermöglicht wird, fremdes oder eigenes Handeln bewusst wahrzunehmen, zu reflektieren und daraus einen Erkenntnisgewinn zu ziehen (Janik et al. 2013, 66), ohne dass dabei ein Handlungsdruck besteht (Riegel 2013, 15).

Im begleitenden Forschungsprojekt Nawi-In werden die Reflexionen inhaltsanalytisch (Kuckartz 2016) auf die Kompetenzen der Studierenden für inklusiven naturwissenschaftlichen Unterricht ausgewertet, Kompetenzprofile von Studierenden beider Schulstufen (Primar- und Sekundarstufe I) erstellt und verglichen. Im dritten Semester werden die Projekte von den Studierenden ausgewertet und ein Bericht erstellt, der die Videoreflexionen umfasst. Die Begleitforschung steht noch aus.

\section{Zusammenfassung und Ausblick}

Inklusion in der Lehrer*innenbildung kann in der heutigen Zeit nicht mehr als Nebensache behandelt werden, sondern muss als Schwerpunkt und dauerhaft in allen Bildungsbereichen verankert werden. Die Professionalisierung von angehenden Lehrkräften zur Vorbereitung auf schulische Inklusion ist eine Gemeinschaftsaufgabe von Forschung und Lehre. Vor allem die Fachdidaktiken sind hier in der Pflicht, exklusive Mechanismen in ihren Fächern zu identifizieren und die Aufbereitung ihrer speziellen Fachinhalte didaktisch inklusiv auszugestalten. Da im jetzigen Schulsystem in Deutschland keine Transdisziplinarität der einzelnen Fächer durch eine Auflösung von Fächergrenzen zu erwarten ist, muss der Weg über eine inklusive Ausgestaltung in den einzelnen Fächern gewählt werden. Einige Hochschulen haben sich bereits auf den 
Weg gemacht, Inklusion als Querschnittskompetenz systematisch in die Lehre zu integrieren und sich über Forschungsprojekte in diesem Themenbereich zu vernetzen. Beispielsweise findet im Förderprogramm der empirischen Bildungsforschung »Fachkräftequalifizierung für inklusive Bildung « des BMBF (2016) ein enger Austausch zwischen den teilnehmenden Hochschulen statt.

Es existieren bereits Lehr-/Lernformate, die sich für eine Umsetzung inklusiven Unterrichts in der schulischen Praxis eignen, wie am Beispiel der Naturwissenschaften aufgezeigt wurde. Die gezielte Förderung der Professionalisierung angehender Lehrkräfte im Planen und Umsetzen von inklusivem naturwissenschaftlichen Unterricht einerseits und die Beforschung dieser Professionalisierung in der unterrichtlichen Praxis andererseits soll die Lehramtsstudierenden bei ihrer Kompetenzentwicklung unterstützen und die Ergebnisse der Begleitforschung zur hochschuldidaktischen Entwicklung beitragen. Hierfür werden vor allem Unterrichtsvideos genutzt, die sowohl von erfahrenen Lehrkräften in Form von Fremdvideos vorliegen als auch in Form von Eigenvideos von den Studierenden selbst. Die Studierenden nutzen die Videos zur Reflexion und der Entwicklung professioneller Kompetenz für inklusiven Fachunterricht. Die Videos dienen der Forschung zur Analyse der Kompetenzentwicklung und zur Identifizierung von Gelingensbedingungen inklusiv gestalteten naturwissenschaftlichen Unterrichts. In diesem Forschungszyklus nimmt die Videografie eine wichtige Rolle ein, da sie unterrichtliche Praxis abbildet und Prozesse des unterrichtlichen Handelns analysierbar macht.

Forschung und Lehre an den Hochschulen sind insgesamt auf dem Weg, sich hin zu einer inklusiven Ausrichtung in der Professionalisierung angehender Lehrkräfte zu bewegen. Für die Fachdidaktiken ist hier nach wie vor Nachholbedarf zu verzeichnen, damit inklusiver Fachunterricht zur Selbstverständlichkeit wird und angehende Lehrpersonen in ihrer Kompetenzentwicklung diesbezüglich unterstützt werden.

\section{Anmerkung}

1 Das Projekt Nawi-In wird vom BMBF (Förderkennzeichen 01NV1731, Laufzeit 01.04.2018 -31.03.2021) gefördert.

\section{Literatur}

Abels, Simone. 2016. „Chemieunterricht und Inklusion - zwei unvereinbare Kulturen?« In Befähigung zu gesellschaftlicher Teilhabe: Beiträge der fachdidaktischen Forschung, herausgegeben von Jürgen Menthe, Dietmar Höttecke, Thomas Zabka, Marcus Hammann und Martin Rothgangel, 323-334. Münster: Waxmann.

Abels, Simone. 2019. »Potentialorientierter Naturwissenschaftsunterricht«. In Potenzialorientierte Förderung in den Fachdidaktiken, herausgegeben von Marcel Veber, Ralf Benölken und Michael Pfitzner, 61-78. Münster: Waxmann. 
Abels, Simone. 2019. » Science Teacher Professional Development for Inclusive Practice.« International Journal of Physics and Chemistry Education 11: 19-30.

Abels, Simone, und Silvija Markic. 2013. »Umgang mit Vielfalt: neue Perspektiven im Chemieunterricht." Naturwissenschaften im Unterricht: Chemie 24: 2-6.

Abels, Simone, und Elisabeth Minnerop-Haeler. 2015. »Lernwerkstatt `Mensch «: von den Fragen der Schülerlnnen ausgehen.«Praxis Schule 6: 26-33.

Abels, Simone und Sandra Schütz. 2016. »Fachdidaktik trifft inklusive Pädagogik - (Unausgeschöpfte) Potentiale in der Lehrerbildung." Zeitschrift für Heilpädagogik 67: 425-436.

Ainscow, Mel, Tony Booth und Alan Dyson. 2006. »Inclusion and the standards agenda: negotiating policy pressures in England.«International Journal of Inclusive Education 10: 295-308.

Alsawaie, Othman N., und Iman M. Alghazo. 2010. »The effect of video-based approach on prospective teachers' ability to analyze mathematics teaching." Journal of Mathematics Teacher Education 13: 223-241.

Amrhein, Bettina, und Kersten Reich. 2014. »Inklusive Fachdidaktik.« In Fachdidaktik inklusiv: Auf der Suche nach didaktischen Leitlinien für den Umgang mit Vielfalt in der Schule, herausgegeben von Bettina Amrhein und Myrle Dziak-Mahler, 31-44. Münster: Waxmann.

Andersson, Kristina, und Annica Gullberg. 2014. "What is science in preschool and what do teachers have to know to empower children?« Cultural Studies of Science Education 9, 2: 275-296.

Baumert, Jürgen, und Mareike Kunter. 2006. „Stichwort: Professionelle Kompetenz von Lehrkräften."Zeitschrift für Erziehungswissenschaft 9: 469-520.

Booth, Tony. 2014. "Structuring Knowledge for all in the 21st Century.«In Fachdidaktik inklusiv: Auf der Suche nach didaktischen Leitlinien für den Umgang mit Vielfalt in der Schule, herausgegeben von Bettina Amrhein und Myrle Dziak-Mahler, 57-69. Münster: Waxmann.

Brauns, Sarah, Daniela Egger und Simone Abels. In Vorbereitung. »Forschendes Lernen auf Hochschul- und Unterrichtsebene beforschen«.

Brauns, Sarah, Daniela Egger, Matthias Barth und Simone Abels. In Vorbereitung. »Prädiktoren gelingenden inklusiven Naturwissenschaftsunterrichts - ein Review«.

Brigham, Frederick, Thomas Scruggs und Margo Mastropieri. 2011. "Science Education and Students with Learning Disabilities. « Learning Disabilities Research \& Practice 26, 4: 223-232.

European Agency for Development in Special Needs Education. 2012. Teacher Education for Inclusion: Profile of Inclusive Teachers. Zugriff 03.04.2019. https://www.european-agency.org/sites/ default/files/Profile-of-Inclusive-Teachers.pdf.

Feyerer, Ewald. 2004. »Inklusion: Herausforderung an die Lehre am Beispiel der Pädagogischen Akademie des Bundes in Linz." In Inklusive Pädagogik, herausgegeben von Irmtraud Schnell und Alfred Sander, 339-349. Bad Heilbrunn/Obb.: Klinkhardt.

Feyerer, Ewald. 2012. »Allgemeine Qualitätskriterien inklusiver Pädagogik und Didaktik. Zeitschrift für Inklusion 0, 3: o. S. Zugriff 21.11.2016. http://www.inklusion-online.net/index.php/ inklusion-online/article/view/51/51

Fichten, Wolfgang, Ulf Gebken und Alexandra Obolenski. 2006. »Konzeption und Praxis der Oldenburger Teamforschung.« In Forschendes Lernen: Theorie und Praxis einer professionellen Lehrerlnnenausbildung, herausgegeben von Alexandra Obolenski und Hilbert Meyer, 131-151. Oldenburg: Universität Oldenburg.

Fink, Arlene. 2009. Conducting Research Literature Reviews - From the Internet to Paper. California: SAGE Publications.

Gilbert, Jonathan. 2006. »On the Nature of »Context« in Chemical Education.« International Journal of Science Education 28, 9: 957-976.

Goodwin, Charles. 1994. Professional vision. American Anthropologist 96, 3: 606-633.

Greiten, Silvia. 2014. »Welche Kompetenzen für die Unterrichtsplanung benötigen LehrerInnen 
an Regelschulen für einen inklusiven, auf individuelle Förderung ausgerichteten Unterricht? Erste Ergebnisse aus einer qualitativ-empirischen Studie." In Inklusive Bildung: Erkenntnisse und Konzepte aus Fachdidaktik und Sonderpädagogik, herausgegeben von Silke Trumpa, Stefanie Seifried, Eva Franz und Theo Klauß, 107-121. Weinheim und Basel: Beltz Juventa.

Gröschner, Alexander, Susi Klaß und Matthias Dehne. 2018. »Effekte des videobasierten peer-coaching auf die Kompetenzeinschätzung."Zeitschrift für Hochschulentwicklung 13, 1: 45-68.

Gudjonsdottir, Hafdis, und Edda Óskarsdóttir. 2016. »Inclusive education, pedagogy and practice.» Science Education towards Inclusion 22: 1-16.

Hattie, John. 2003. »Teachers Make a Difference, What is the research evidence? «In Building Teacher Quality: What does the research tell us? Melbourne: Australian Council for Educational Research (ACER): 1-17. Zugriff 30.03.2019. https://research.acer.edu.au/cgi/viewcontent.cgi? article $=1003 \&$ context $=$ research_conference_2003.

Hoeks, Marrit. 2011. "Lernen mit einem Videoportfolio in der Lehrerausbildung." Fremdsprache Deutsch 45: 53-56.

Huber, Ludwig. 2006. »Forschendes Lernen in deutschen Hochschulen. Zum Stand der Diskussion." In Forschendes Lernen: Theorie und Praxis einer professionellen LehrerInnenausbildung, herausgegeben von Alexandra Obolenski und Hilbert Meyer, 15-36. Oldenburg: Universität Oldenburg.

Janik, Tomas, Eva Minarikova und Petr Najvar. 2013. „Der Einsatz von Videotechnik in der Lehrerbildung: Eine Übersicht leitender Ansätze.» In Videobasierte Kompetenzforschung in den Fachdidaktiken, herausgegeben von Ulrich Riegel und Klaus Macha, 63-78. Münster: Waxmann.

Kahlert, Joachim. 2009. Der Sachunterricht und seine Didaktik. Bad Heilbrunn: UTB.

Kuckartz, Udo. 2016. Qualitative Inhaltsanalyse. Methoden, Praxis, Computerunterstützung (Grundlagentexte Methoden, 3., überarbeitete Auflage). Weinheim: Beltz Juventa.

Kunter, Mareike, Uta Klusmann und Jürgen Baumert. 2009. »Professionelle Kompetenz von Mathematiklehrkräften: Das COACTIV-Modell.« In Lehrprofessionalität - Bedingungen, Genese, Wirkungen und ihre Messung, herausgegeben von Olga Zlatkin-Troitschanskaia, Klaus Beck, Detlef Sembill, Nickolaus Reinhold und Regina Mulder, 153-165. Weinheim: Beltz.

Kultusministerkonferenz. 2011. »Inklusive Bildung von Kindern und Jugendlichen mit Behinderungen in Schulen.« Zugriff 01.06.2019. https://www.kmk.org/fileadmin/Dateien/veroeffentlich ungen_beschluesse/2011/2011_10_20-Inklusive-Bildung.pdf.

Leonhard, Tobias, und Simone Abels. 2017. »Der reflective practitioner: Leitfigur oder Kategorienfehler einer reflexiven Lehrerinnen- und Lehrerbildung?« In Reflexive Lehrerbildung revisited: Traditionen - Zugänge - Perspektiven, herausgegeben von Constanze Berndt, Thomas Häcker und Tobias Leonhard, 46-55. Bad Heilbrunn: Julius Klinkhardt.

Lindmeier, Anke. 2013. „Video-vignettenbasierte standardisierte Erhebung von Lehrerkognitionen. "In Videobasierte Kompetenzforschung in den Fachdidaktiken, herausgegeben von Ulrich Riegel und Klaus Macha, 45-62. Münster: Waxmann.

Loucks-Horsley, Susan, Nancy Love, Katherine E. Stiles, Susan Mundry und Peter E. Hewson. 2003. Designing professional development for teachers of science and mathematics. USA: Corwin Press.

Markic, Silvija, und Simone Abels. 2014. »Heterogeneity and Diversity: A Growing Challenge or Enrichment for Science Education in German Schools?«EURASIA Journal of Mathematics, Science and Technology Education 10, 4: 271-283.

Menthe, Jürgen, Simone Abels, Eva Blumberg, Theresa Fromme, Annette Marohn, Andreas Nehring und Lisa Rott. 2017. »Netzwerk inklusiver naturwissenschaftlicher Unterricht.« In Implementation fachdidaktischer Innovation im Spiegel von Forschung und Praxis. Gesellschaft für Didaktik 
der Chemie und Physik, herausgegeben von Christian Maurer, 800-802. Universität Regensburg.

Menthe, Jürgen, und Thomas Hoffmann. 2015. »Inklusiver Chemieunterricht: Chancen und Herausforderungen. "In Inklusiver Fachunterricht in der Sekundarstufe, herausgegeben von Judith Riegert und Oliver Musenberg, 131-141. Stuttgart: Kohlhammer.

Moser, Vera, und Andreas Kropp. 2014. »Abschlussbericht: Kompetenzen in inklusiven settings (KIS) - Vorarbeiten zu einem Kompetenzstrukturmodell sonderpädagogischer Lehrkräfte.« Zugriff 30.05.2019. https://www.kmk.org/fileadmin/Dateien/veroeffentlichungen_beschlue sse/2011/2011_10_20-Inklusive-Bildung.pdf.

Musenberg, Oliver, und Riegert Judith. 2015. »Inklusiver Fachunterricht als didaktische Herausforderung. «In Inklusiver Fachunterricht in der Sekundarstufe, herausgegeben von Judith Riegert und Oliver Musenberg, 13-28. Stuttgart: Kohlhammer.

Niedersächsisches Kultusministerium. o.J. „GHR 300«. Zugriff 10.04.2019. https://www.mk. niedersachsen.de/startseite/schule/lehrkraefte/studium_master_ghr_300/ghr-300 --101533.html.

Obolenski, Alexandra. 2004. »Qualifizierung für eine inklusive Pädagogik: Anforderungen an die LehrerInnenbildung.«In Inklusive Pädagogik, herausgegeben von Irmtraud Schnell \& Alfred Sander, 313-319. Bad Heilbrunn/Obb.: Klinkhardt.

Pädagogische Hochschule Heidelberg. 2019. „Übergreifender Studienbereich (ÜSB).« Zugriff 09.04.2019. https://www.ph-heidelberg.de/uesb/informationen/po-2011/ziele.html.

Riebling, Linda. 2013. Sprachbildung im naturwissenschaftlichen Unterricht: Eine Studie im Kontext migrationsbedingter sprachlicher Heterogenität. Münster: Waxmann.

Riegel, Ulrich. 2013. »Videobasierte Kompetenzforschung in den Fachdidaktiken: Einleitung.« In Videobasierte Kompetenzforschung in den Fachdidaktiken, herausgegeben von Ulrich Riegel und Klaas Macha, 9-24. Münster: Waxmann.

Sawalies, Jennifer, Marcel Veber, David Rott und Christian Fischer. 2013. »Inklusionspädagogik in der ersten Phase der Lehrerbildung: Eine explorative Studie zu Stand und Unterschieden universitärer Lehrangebote für die Regelschullehrämter.« In Schulpädagogik heute 4, 8: 1-17.

Schmidkunz, Heinz, und Helmut Lindemann. 2003. Das forschend-entwickelnde Unterrichtsverfahren: Problemlösen im naturwissenschaftlichen Unterricht. Hohenwarsleben: Westarp Science.

Schönig, Wolfgang, und John A. Fuchs. 2016. »Inklusion inkludiert: Was bedeutet Inklusion 10 Jahre nach der UN-BRK? «In Inklusion: Gefordert! Gefördert?: Schultheoretische, raumtheoretische und didaktische Zugänge, herausgegeben von Wolfgang Schönig und John A. Fuchs, 9-31. Bad Heilbrunn: Klinkhardt.

Seidel, Tina, Manfred Prenzel und Mareike Kobarg (Hrsg.). 2005. How to run a video study: Technical report of the IPN Video Study. Münster: Waxmann.

Seidel, Tina, Kathleen Stürmer, Geraldine Blomberg, Mareike Kobarg und Katharina Schwindt. 2011. »Teacher learning from analysis of videotaped classroom situations: Does it make a difference whether teachers observe their own teaching or that of others? « Teaching and Teacher Education 27: 259-267.

Seitz, Simone. 2011. »Eigentlich nichts Besonderes - Lehrkräfte für die inklusive Schule ausbilden.« Zeitschrift Für Inklusion 3: o.S. Zugriff 03.06.2015. http://www.inklusion-online.net/ index.php/inklusion-online/article/view/83/83.

Sherin, Miriam. 2007. „The Development of Teacher's Professional Vision in Video Clubs.« In Video Research in the Learning Sciences, herausgegeben von Ricki Goldman, Roy Pea und Sharon J. Derry, 383-396. Mahwah: Lawrence Earlbaum.

Shulman, Lee S. 1986. »Those Who Understand: Knowledge Growth in Teaching."Educational Researcher 15: 4-14. 
Stroh, Michael. 2015. »Inklusion im naturwissenschaftlichen Unterricht - Beschreibung eines Spannungsfeldes." In Grundlagen inklusiver Bildung. Teil 1. Inklusive Unterrichtspraxis und -entwicklung, herausgegeben von Catrin Seidenbiedel und Caroline Theurer, 110-124. Immenhausen bei Kassel: Prolog.

Therrien, William, Jonte Taylor, John Hosp, Erica Kaldenberg und Jay Gorsh. 2011. »Science Instruction for Students with Learning Disabilities: A Meta-Analysis." Learning Disabilities Research \& Practice, 26, 4: 188-203.

Troll, Bianka, Michael Besser, Simone Abels, Michael Ahlers, Dominik Leiss und Jessica Süßenbach. 2019. »Preparing pre-service teachers for inclusive education: Analyzing the effect of different types of subject-specific learning opportunities at university on beliefs, self-efficacy and pedagogical content knowledge." In Inclusive mathematics education: Research results from Brazil and Germany, herausgegeben von Michael Knigge, David Kollosche, Ole Skovsmose, Renato Marcone und Miriam Godoy Penteado, 537-559. Wiesbaden: Springer VS.

UNESCO. 2009. »Inklusion: Leitlinien für die Bildungspolitik.« Zugriff 02.04.2019. https://www. unesco.de/sites/default/files/2018-05/2014_Leitlinien_inklusive_Bildung.pdf.

UNESCO. 2015. »UNESCO: Weltbildungsbericht 2015.«Zugriff 12.04.2019. https://www.unesco.de/ bildung/bildungsagenda-2030/unesco-weltbildungsbericht/bildungsagenda-2030.

United Nations. 2006. "Convention on the Rights of Persons with Disabilities.« Zugriff 02.04.2019. https://www.un.org/development/desa/disabilities/convention-on-the-rights-of-persons -with-disabilities/article-24-education.html.

van Es, Elizabeth A., und Miriam G. Sherin. 2008. »Mathematics teachers' >learning to notices in the context of a video club." Teaching and Teacher Education 24, 2: 244-276.

Villanueva, Mary, Jonte Taylor, William Therrien und Brian Hand. 2012. "Science education for students with special needs."Studies in Science Education, 48, 2: 187-215.

Weinert, Franz E. 2001. „Vergleichende Leistungsmessung in Schulen: eine umstrittene Selbstverständlichkeit.« In Leistungsmessungen in Schulen, herausgegeben von Franz E. Weinert, 17-31. Weinheim: Beltz.

Weishaupt, Horst. 2015. »Aus-, Fort- und Weiterbildung für ein Schulwesen auf dem Weg zur inklusiven Schule.« Zeitschrift für Heilpädagogik 66, 5: 216-229.

Wendt, Heike, Wilfried Bos, Christopher Selter, Olaf Köller, Knut Schwippert und Daniel Kasper (Hrsg.). 2016. TIMSS 2015: Mathematische und naturwissenschaftliche Kompetenzen von Grundschulkindern in Deutschland im internationalen Vergleich. Münster: Waxmann.

Werning, Rolf, und Meltem Avci-Werning. 2015. Herausforderung Inklusion in Schule und Unterricht: Grundlagen, Erfahrungen, Handlungsperspektiven. Seelze: Kallmeyer.

WiFF (Weiterbildungsinitiative Frühpädagogische Fachkräfte). 2013. Inklusion - Kinder mit Behinderung. Grundlagen für die kompetenzorientierte Weiterbildung. München: WiFF Wegweiser Weiterbildung.

Wocken, Hans. 2014. Das Haus der inklusiven Schule: Baustellen - Baupläne - Bausteine. Hamburg: Feldhaus.

WWU Münster. 2019. »Projektbeschreibung.« Zugriff 30.05.2019. https://www.uni-muenster.de/ Koviu/Projekt/projektbeschreibung.html.

Ziemen, Kerstin. 2014. »Inklusion und deren Herausforderungen für die (Fach-)Didaktik.«In Fachdidaktik inklusiv: Auf der Suche nach didaktischen Leitlinien für den Umgang mit Vielfalt in der Schule, herausgegeben von Bettina Amrhein und Myrle Dziak-Mahler, 45-55. Münster: Waxmann.

ZZL (Zukunftszentrum Lehrerbildung). 2018. »Zwischenbilanz 2018 - ZZL-Netzwerk.» Zugriff 03.04.2019. https://www.leuphana.de/fileadmin/user_upload/Forschungseinrichtungen/zzl /files/ZZL/Zwischenbilanz_2018_ZZL-Netzwerk_30.08.2018.pdf. 


\section{Die Autorlnnen}

Daniela Egger arbeitet als wissenschaftliche Mitarbeiterin am Institut für Nachhaltige Chemie und Umweltchemie (INUC) im Bereich Didaktik der Naturwissenschaften an der Leuphana Universität Lüneburg. Ihre Arbeitsschwerpunkte sind Inklusiver naturwissenschaftlicher Unterricht und Lehrer*innenprofessionalisierung. Seit ihrem Abschluss Master of Education (2018) ist sie Promovierende im Rahmen des Projekts Nawi-In.

Kontakt: daniela.egger@leuphana.de

Sarah Brauns ist seit ihrem Abschluss des Master of Education (2018) wissenschaftliche Mitarbeiterin und Doktorandin am Institut für Nachhaltige Chemie und Umweltchemie (INUC) in der Arbeitsgruppe Didaktik der Naturwissenschaften an der Leuphana Universität Lüneburg im Projekt Nawi-In.

Kontakt: sarah.brauns@leuphana.de

Katja Sellin, wissenschaftliche Mitarbeiterin und Doktorandin, an der Leuphana Universität Lüneburg, arbeitet am Institut für integrative Studien (INFIS), Sachunterricht und Bildung für eine nachhaltige Entwicklung und ist ebenfalls Mitarbeiterin im Projekt Nawi-In.

Kontakt: katja.sellin@leuphana.de

Matthias Barth, Prof. Dr., ist stellvertretender Projektleiter von Nawi-In am Institut für integrative Studien (INFIS). Seine Forschungsschwerpunkte sind Sachunterricht und Bildung für eine nachhaltige Entwicklung (BNE), Inklusiver naturwissenschaftlicher Unterricht und Lehrer*innenprofessionalisierung.

Kontakt: matthias.barth@leuphana.de

Simone Abels, Prof. Dr., ist Projektleiterin von Nawi-In und arbeitet seit dem Jahr 2016 am Institut für Nachhaltige Chemie und Umweltchemie (INUC) an der Leuphana Universität Lüneburg. Sie ist Professorin für Didaktik der Naturwissenschaften. Ihre Forschungsgebiete sind Forschendes Lernen, Inklusiver naturwissenschaftlicher Unterricht und reflexive Lehrer*innenbildung.

Kontakt:simone.abels@leuphana.de 